\title{
Proper motions of open clusters based on the TYCHO2 Catalogue
}

\section{Clusters farther than $1 \mathrm{kpc}^{\star, \star \star}$}

\author{
W. S. Dias, J. R. D. Lépine, and B. S. Alessi \\ Universidade de São Paulo, Dept. de Astronomia, CP 3386, São Paulo 01060-970, Brazil
}

Received 26 February 2002 / Accepted 19 March 2002

\begin{abstract}
We determined the mean absolute proper motion of 94 open clusters situated farther than $1 \mathrm{kpc}$ from the Sun. The results are derived from the stellar proper motion data given in the Tycho2 Catalogue. The mean proper motion of the clusters and membership probability of individual stars were obtained from the proper motion data by applying the statistical method proposed by Sanders (1971). The measurements made use of a large number of stars, usually several tens, for each cluster. The total number of stars investigated in the fields of the clusters is 4864 of which 2021 were considered members. For 55 clusters, this is the first determination of the proper motion.
\end{abstract}

Key words. Galaxy: open clusters and association: general

\section{Introduction}

Proper motions of stars in the field of open clusters are fundamental to segregate their members. The common origin of the stars of a cluster suggests that they present a common motion, and the information on proper motion and radial velocity can be used in this identification task.

The knowledge of the members of the clusters is essential for different studies: photometric investigations (Pinsonneault 1998), studies of internal motions and dynamics of the stars in the clusters (Perryman et al. 1998), determination of the IMF (Sanner et al. 2001) and of the luminosity function (Belikov et al. 2000).

On the other hand, the mean proper motions can be useful in the study of Galactic orbits and in Galactic structure investigations (Lynga \& Palous 1987).

The aim of this work is to determine the absolute proper motion of the clusters using a large number of stars for each one.

Send offprint requests to: W. S. Dias,

e-mail: wilton@iagusp.usp.br

* Based on observations of the ESA Hipparcos satellite.

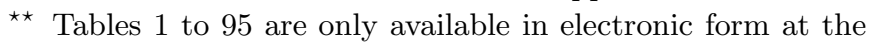
CDS via anonymous ftp to

cdsarc.u-strasbg.fr $(130.79 .128 .5)$ or via

http://cdsweb.u-strasbg.fr/cgi-bin/qcat?J/A+A/388/168
In Paper I we determined the absolute proper motion of 112 open clusters situated within $1 \mathrm{kpc}$ from the Sun (Dias et al. 2001). In this second paper, the objective was to determine the absolute proper motions of all the catalogued clusters farther than $1 \mathrm{kpc}$.

The results are incorporated in a new version of open clusters catalogue (Dias et al. 2002, hereinafter DAML2002) that presents the fundamental parameters and kinematic data for 1537 objects.

In Sect. 2 we describe the method and the procedures adopted in this investigation. Section 3 presents the main results and their comparison with those given in the literature.

\section{Determination of the mean absolute proper motions}

Following the procedure described in Paper I, we selected in the DAML2002 database ${ }^{1}$ all the clusters catalogued with distance greater than $1 \mathrm{kpc}$.

We searched for the selected clusters in the Tycho2 Catalogue (Hog et al. 2000), using the central coordinates and the radius taken from the database. All the stars situated within the limits of the clusters were investigated. For open clusters with an apparent diameter less than $15^{\prime}$

\footnotetext{
1 Available at http://www.iagusp.usp.br/〜 wilton/
} 
we searched for stars in an area 4 times the cluster area, and if $D \geq 15^{\prime}$ we used the area covered by the cluster.

In Paper I we argued that the Tycho2 Catalogue is the main source of data, at least in large scale, to be used in this kind of investigation (see Dias et al. 2001; Sanner et al. 2001). However it is important to note that because of its limiting magnitude (about $13 \mathrm{mag}$ ), this catalogue is not very dense. This is the reason why it is specially difficult to obtain satisfactory results for open clusters with $D \leq 6^{\prime}$. Actually, we were able to determine the mean proper motion of 94 clusters, which is only about $40 \%$ of the total investigated.

When a sufficient number of stars was found in the region of the cluster, we performed the statistical analysis of Sanders (1971) to segregate field and clusters stars.

The method fits a two-dimensional Gaussian distribution to the vector point diagram (VPD) and the best parameters are determined by a maximum likelihood procedure. The details are given by Sanders (1971), and discussions on improvements of the method, can be found in Zhao \& He (1990).

\section{Results and discussions}

Table 1 (available in electronic form) presents our final results for the clusters and fields parameters, determined by the method of Sanders. The mean proper motion of the studied open clusters are also available in DAML2002. Tables 2 to 95, only available in electronic form, list the stars in the limits of each cluster, with the membership probabilities calculated by our method.

As performed in Paper I, we checked our mean absolute proper motions against the mean proper motions derived by BDW and other recent papers. In a comparison with BDW's results we found 39 common objects. A Gaussian fit to the differences (Fig. 1) gives a mean proper motion difference of -0.6 in $\mu_{\alpha} \cos \delta$ and -0.1 in $\mu_{\delta}$; the standard deviations are $1.6 \mathrm{mas} / \mathrm{yr}$ in $\mu_{\alpha} \cos \delta$ and $1.8 \mathrm{mas} / \mathrm{yr}$ in $\mu_{\delta}$.

\subsection{NGC 2360, NGC 2244, NGC 6531 and Trumpler 15}

A few open clusters marked in Fig. 1 present differences with BDW greater than the maximum expected value (about $2.5 \mathrm{mas} / \mathrm{yr}$, which is the typical error in Tycho2 proper motions of single stars).

In the cases of the clusters NGC 2360, NGC 2244, NGC 6531 and Trumpler 15 it seems that our method is unable to distinguish the members from the background, which has approximately the same proper motion (see the VPDs in Fig. 2). Based on the bad segregation of the two populations (field and cluster) in the VPD, we suggest that the results given by BDW are the correct ones.

However, it is important to pay close attention to the fact that the proper motions provided by the Tycho 2 catalogue differs from the Hipparcos ones, specially for the stars used by BDW for the cluster NGC 6531.

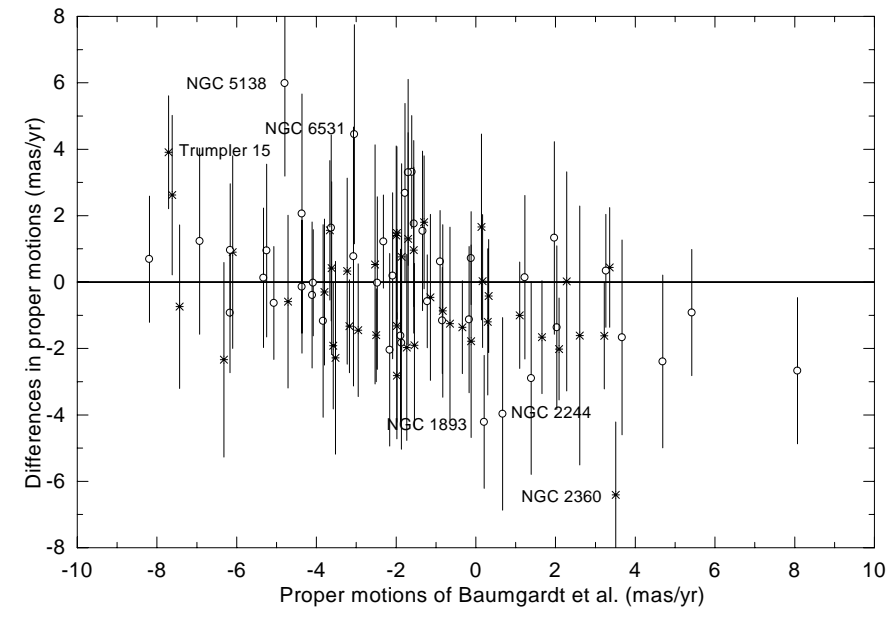

Fig. 1. Comparison of our mean proper motions with those determined by BWD. The differences are presented as a function of the results of BWD. The open circles represent the values in $\mu_{\alpha} \cos \delta$, and the stars in $\mu_{\delta}$.

\subsection{NGC 1893 and NGC 5138}

The proper motion provided by BDW for these two clusters were based on only one star.

For the NGC 1893 the star considered is HIP 25103 $\left(\mu_{\alpha} \cos \delta=-1.3 \mathrm{mas} / \mathrm{yr}\right.$ and $\left.\mu_{\delta}=+0.21 \mathrm{mas} / \mathrm{yr}\right)$. This star is present in the Tycho2 catalogue $\left(\mu_{\alpha} \cos \delta=\right.$ $-1.1 \mathrm{mas} / \mathrm{yr}$ and $\left.\mu_{\delta}=-1.8 \mathrm{mas} / \mathrm{yr}\right)$, so that the proper motion is a little different from the Hipparcos one in $\mu_{\delta}$. Note that the radial velocity of HIP $25103(V=$ $-7.5 \mathrm{~km} \mathrm{~s}^{-1}$, Crampton \& Fisher 1974) is compatible with the mean radial velocity of the cluster $\left(-4.7 \pm 4.4 \mathrm{~km} \mathrm{~s}^{-1}\right)$ provided by Rastorguev et al. (1999). From a photometric point of view, the spectral type of this star (O5) suggests that it belongs to the cluster.

The difference found in $\mu_{\delta}$ for that star explains partly the difference in the mean proper motion of the cluster in the two works. Since we used a dozen stars to segregate the members, we believe that our result is the more correct one, considering also that our result maintains the membership of the star HIP 25103.

For the NGC 5138 the star considered by BDW is HIP 65618. This star is present in the Tycho2 sample and our analysis shows that this star has high membership probability $(P=85 \%)$. The difference obtained is due to the difference between Tycho2 $\left(\mu_{\alpha} \cos \delta=-3.00\right.$ mas $/ y r$ and $\left.\mu_{\delta}=+3.10 \mathrm{mas} / \mathrm{yr}\right)$ and Hipparcos $\left(\mu_{\alpha} \cos \delta=\right.$ $-1.73 \mathrm{mas} / \mathrm{yr}$ and $\left.\mu_{\delta}=-4.79 \mathrm{mas} / \mathrm{yr}\right)$ proper motions. To corroborate that our result is correct we note that Dias (2000) determined the mean proper motion of this cluster, obtaining components $\left(\mu_{\alpha} \cos \delta=-4.2 \mathrm{mas} / \mathrm{yr}\right.$ and $\left.\mu_{\delta}=+1.9 \mathrm{mas} / \mathrm{yr}\right)$ compatible with those derived in the present paper.

Based on this analysis we suggest that our proper motion determinations, which rely on a large number of 

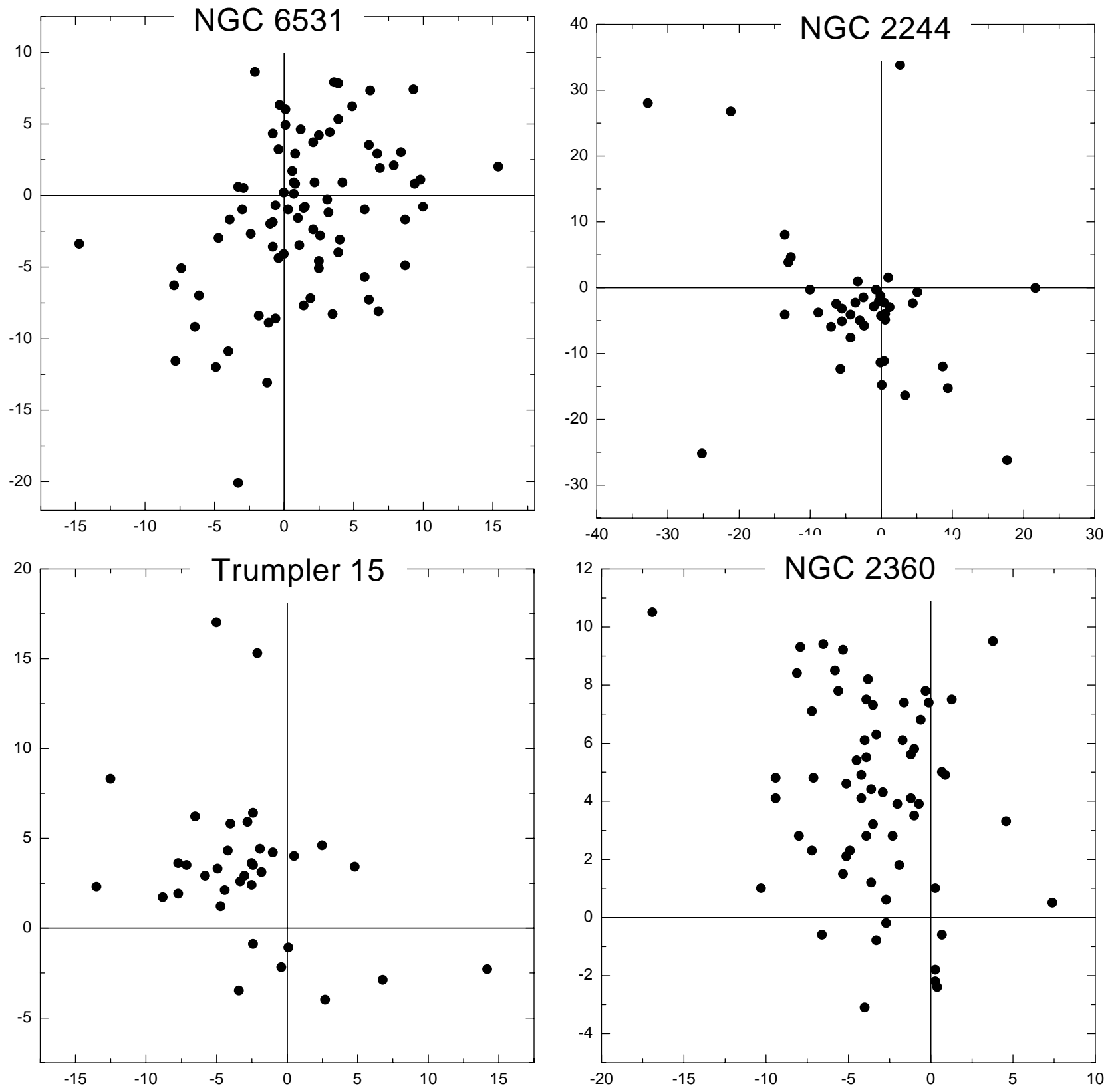

Fig. 2. Vector proper motion diagram of the stars in the region of the open clusters NGC 2360, NGC 2244, NGC 6531 and Trumpler 15. The axes present $\mu_{\alpha} \cos \delta$ and $\mu_{\delta}$, in mas/yr.

stars, are the correct ones for the clusters NGC 1893 and NGC 5138.

\section{Summary}

Mean absolute proper motions of 94 open clusters from all over the sky, farther than $1 \mathrm{kpc}$, were determined using the data provided by the Tycho2 Catalogue. Applying the statistical method of Sanders (1971) we asserted the membership of 2021 stars and we determined for the first time mean proper motions of 55 clusters.

A comparison of our results with those of BDW showed that both sets are in agreement. In a few cases with discrepant results NGC 2360, NGC 2244, NGC 6531 and Trumpler 15, we suggest that the results from BDW are the correct ones. However for the clusters NGC 1893 and NGC 5138 we could show that the differences were real differences between Tycho2 and Hipparcos, or due to the too small numbers of stars considered by BDW. The comparison makes us confident that the proper motions derived for the first time are correct, and provide a useful confirmation of BDW results, using a larger sample of stars.

Acknowledgements. Extensive use has been made of the SIMBAD and BDA databases. This project is supported by FAPESP (grant number 99/11781-4). 


\section{References}

Baumgardt, H., Dettbarn, C., \& Wielen, R. 2000, A\&AS, 146, 251 (BDW).

Belikov, A. N., kharchenko, N. V., Piskunov, A. E., \& Schilbach, E. 2000, A\&A, 358, 886

Crampton, D., \& Fisher, W. A. 1974, PDAO, 14, 283

Dias, W. S. 2000, Master dissertation: Proper motion and membership in open clusters, Universidade de São Paulo, São Paulo, Brazil

Dias W. S., Lépine J. R. D., \& Alessi B. S. 2001, A\&A, 376, 441

Dias, W. S., Alessi, B. S., Moitinho, A., \& Lépine, J. R. D. 2002, A\&A, accepted

ESA, 1997, The Hipparcos and TYCHO Catalogues, ESA SP1200
Hog, E., Fabricius, C., Makarov, V. V., et al. 2000, A\&A, 355,27

Lynga, G., \& Palous, J. 1987, A\&A, 188, 35

Perryman, M. A. C., Brown, A. G. A., Lebreton, Y., et al. 1998, A\&A, 331, 81

Pinsonneault, M. H., Stauffer, J., Soderblom, D. R., King, J. R., \& Hanson, R. B. 1998, ApJ, 504, 170

Rastorguev, A. S., Glushkova, E. V., Danbis, A. K., \& Zabolotskikh, M. V. 1999, Astron. Lett., 25, 68

Sanders, W. L. 1971, A\&A, 14, 226

Sanner, J., Brunzendorf, J., Will, J.-M., \& Geffert, M. 2001, A\&A, 369, 511

Zhao, J. L., \& He, Y. P. 1990, A\&A, 237, 54 